Original Research Paper

\title{
Expression Pattern of the Orphan Nuclear Receptor, Nurr1, in the Developing Mouse Forelimb and its Relationship to Limb Skeletogenesis and Osteogenesis
}

\author{
${ }^{1}$ Heba A. Ahmed, ${ }^{1}$ Lidia L. Ibrahim, ${ }^{2}$ Desouki A. El Mekkawy and ${ }^{1,3}$ Abeer El Wakil \\ ${ }^{I}$ Department of Biological and Geological Sciences, Faculty of Education, University of Alexandria, Egypt \\ ${ }^{2}$ Department of Zoology, Faculty of Science, University of Alexandria, Egypt \\ ${ }^{3}$ Department of Molecular Biology, Umeå University, Sweden
}

Article history

Received: 28-04-2015

Revised: $27-05-2015$

Accepted: 01-07-2015

Correspondence Author: Abeer El Wakil

Department of Molecular Biology, Umeå University, Sweden

E-mail: abeer_elwakil@yahoo.com

\begin{abstract}
The NR4A orphan nuclear receptor, Nurr1, has been shown to regulate the expression of osteoblastic genes and osteoblastic differentiation. However, the expression profile of Nurr1 in the developing mouse forelimb and its relationship to skeletogenesis has not, to the best of our knowledge, been previously analyzed. In this study, the relationship between Nurr1 expression pattern, skeletogenesis and osteogenesis in the developing mouse forelimb was investigated. The expression level of Nurr1 during development was also quantified by real time-polymerase chain reaction. Our results revealed that Nurr1 is expressed in the mesenchyme cells that will form the skeleton. Nurr1 is aabundantly expressed in the primary ossification centers of the forelimb skeletal elements and its expression level is gradually increased during limb development, particularly, at the onset of ossification. Collectively, these data suggested that Nurrl plays an important role in skeletogenesis and patterning of the developing mouse forelimb.
\end{abstract}

Keywords: Nurr1, Nuclear Orphan Receptors, Osteogenesis, Limb Patterning

\section{Introduction}

Nuclear Receptors (NRs) are DNA binding transcription factors that possess conservative domain organization and control key metabolic and developmental pathways. They are one of the most abundant classes of transcriptional regulators in metazoan animals. They regulate diverse functions such as homeostasis, reproduction, development and metabolism (Laudet and Gronemeyer, 2002). NRs provide direct control of gene expression via various extracellular and intracellular signals. These receptors are subdivided into several subfamilies according to similarities of amino acid sequences and some other properties (Smirnov, 2002). Most of these subfamilies are ligand-inducible transcription factors mediating the effects of small lipophilic ligands such as steroid hormones, retinoids, thyroid hormones and vitamin D. In addition, there are a large number of NRs lacking identified physiological ligands and thus are referred to as orphan nuclear receptors (Giguére, 1999).

Nur-related factor 1 (Nurr1, also known as NR4A2, TINUR, NOT) belongs to the NR4A subfamily of orphan nuclear receptors together with two other members, the nerve growth factor inducible B, (NGFI-B, also known as NR4A1, Nur77, TR3) and neuron-derived orphan receptor 1 (Nor1, NR4A3, MINOR). NGFI-B was found to be induced when cells were treated with the nerve growth factor (Milbrandt, 1988) and Nurr1 and Nor1 were cloned due to their resemblance to other nuclear receptors (Law et al., 1992; Ohkura et al., 1996). Structural analyses of Nurr1 Ligand Binding Domain (LBD) have demonstrated that the ligand-binding pocket of this receptor is filled with bulky sidechains from residues that are conserved in the family implying that the LBD of Nurr1 could not bind any lipophilic ligand in the classical way, in contrast to the other members of the superfamily. Thus, Nurr1 is assumed to regulate gene expression in a ligand independent manner (Wang et al., 2003; Flaig et al., 2005).

During development, Nurr1 is highly expressed in the Central Nervous System (CNS), especially in the Dopaminergic (DA) neurons of the Ventral Midbrain (VMB) (Saucedo-Cardenas and Conneely, 1996; Zetterström et al., 1996a; 1996b). Targeted disruption of Nurr1 gene in Nurr1 null mice arrests the DA neuron precursors development and prevents 
expression of DA neuron specific markers (Zetterström et al., 1997; Castillo et al., 1998). These findings indicate that Nurr1 is involved in neuronal differentiation in developing nervous system. Most studies have focused on Nurrl function in nervous system. However, it is also detected in other sites such as adrenals, liver, bone, synoviocytes and endothelial cells (Murphy et al., 2001; Tetradis et al., 2001a; Honkaniemi et al., 2000; Ohkubo et al., 2002; Liu et al., 2003).

In bone development, Nurr1 has been reported to be expressed in osteoblasts and osteoblastic cell lines (Tetradis et al., 2001a; Lammi et al., 2004). In a study by Lee et al. (2006), Nurrl siRNA was used to downregulate Nurr1 expression in osteoblasts. Reduced expression of Nurr1 resulted in downregulation of the osteoblastic differentiation markers Osteocalcin (OCN) and collagen 1 alpha 1 (Col1A1) and in Reduced Alkaline Phosphatase (ALP) activity. OCN and Col1A1 expression and ALP activity are consequently downregulated in cultured primary osteoblasts from Nurrl null mice. In addition, Nurrl overexpression increased $\mathrm{OCN}$ and Col1 $\mathrm{A} 1$ expression.

Nurrl has been reported in the developing mouse hindlimbs at E11.5 (Zetterström et al., 1996b). In the adult mouse, Nurr1, NGFI-B and Nor1 are expressed in long bones and in calvaria (Pirih et al., 2005). The expression of the members of the NR4A-subfamily are rapidly induced in response to Parathyroid Hormone (PTH) treatment as immediate early genes in primary mouse osteoblasts through the protein kinase A (PKA) pathway in vitro (Tetradis et al. 2001a, 2001b; Pirih et al., 2003). The NR4A family has also been shown to be induced by PTH in vivo in calvariae and long bones when PTH was injected daily to mice (Pirih et al., 2005). In addition to PTH, also other activators of PKA and protein kinase $\mathrm{C}$ (PKC) upregulate the expression of Nurr1 in osteoblasts (Pirih et al., 2004). Regulation of the expression levels of NR4A receptors is most likely an important mechanism modulating their activities.

Although Nurr1 expression has been reported in the developing mouse limb, its expression pattern and its relationship to skeletogenesis have not, to the best of our knowledge, been previously analyzed. So, the main objective of this study is to investigate the expression profile of Nurr1 in the developing mouse forelimb and its relationship to limb patterning and skeletogenesis.

\section{Materials and Methods}

\section{Animals}

Wild type adult CD1 (Swiss) albino mice were purchased from the Medical Research Institute, Alexandria University, Egypt. All mice were kept in a pathogen-free animal room under controlled conditions at $23 \pm 1^{\circ} \mathrm{C}$. The mice were maintained on a $12 \mathrm{~h}$ light-dark cycle with ad libitum access to standard laboratory diet and water. The animals were allowed to acclimatize to the environment for 1 week before the experiment. All experimental protocols were approved by the local council for animal care at Alexandria University and were conducted according to Egyptian laws for animal experimentation.

\section{Histological Examination by Hematoxylin and Eosin $(H \& E)$}

Embryos at E12.5 and E14.5 were collected from the uterus of pregnant females after being anesthetized with isofluorane and dissected. They were washed with Phosphate Buffered Saline (PBS) and forelimbs from E12.5 and E14.5 were collected and fixed overnight in $4 \%$ paraformaldehyde. Each specimen was then washed by PBS and dehydrated in ascending graded ethanol series. After dehydration, the specimens were cleared in xylene, embedded in paraffin wax and serially sectioned at $7 \mu \mathrm{m}$. Slides were deparaffinized, rehydrated, then stained with Ehrlich's hematoxylin and counterstained with eosin for histological examination.

\section{Immunohistochemical Examination of Nurr1}

Paraffin block samples were prepared as the same procedure used in histological examination. 5-7 $\mu \mathrm{m}$ thick sections on polysine slides (Kindler, Freiburg, Germany) were prepared. Slides were deparaffinized in xylol and they were rehydrated through the descending ethanol series. Epitopes were retrieved by 3 min boiling in sodium citrate $10 \mathrm{mM}, \mathrm{pH} 6.0$ at the microwave. Slides were then washed with PBS. For blocking nonspecific background, samples were placed for $15 \mathrm{~min}$ in $5 \%$ bovine serum albumin in PBS with $0.1 \%$ triton. Slides were incubated with a commercial rabbit polyclonal antibody recognizing Nurr1 (dilution 1:100; Santa cruz, USA). Primary antibodies were detected with the secondary antibody coupled to horse radish peroxidase (HRP) (016-030-084, Jackson Immunoresearch, USA) and HRP activity was detected with the chromogenic substrate ImmPACT DAB (SK-4105, Vector Labs, USA).

\section{Microscope Image Acquisition}

Image acquisition was performed with Leica DMD108 digital microimaging microscope (Leica Microsystems, Germany). Objective used was Leica 4 , $\cdot 10, .20$ and $\cdot 40$. Images were minimally processed with ImageJ for global levels corrections.

\section{RNA Extraction and Real Time-Polymerase Chain Reaction (RT-PCR)}

Embryos at E10.5, E12.5, E14.5 and E18.5 were collected from pregnant females into PBS. The forelimb buds and forelimbs were collected from samples, snap-frozen in liquid nitrogen and then transferred to $80^{\circ} \mathrm{C}$. Total RNA was isolated from tissue using the 
classical guanidinium thiocyanate method according to (Chomczynski and Sacchi, 2006). The RNA concentration was measured using the Nanodrop machine and the integrity of RNA was confirmed on agarose gel $1 \%$. First strand cDNA was synthesized using Super Script ${ }^{\mathrm{TM}}$ III Reverse Transcriptase following manufacturer's instructions (Invitrogen, Germany).

RT-qPCR was performed in duplicate for four animals at each stage. The reaction was carried out using SYBR $^{\circledR}$ Green (Qiagen, Hilden, Germany) to monitor double strand DNA synthesis in a LightCycler machine (Roche, Basel, Switzerland). Primers were designed using Probe Finder version 2.49 and have the following sequence: Nurr1 F: 5'- TCA GAG CCC ACG TCG ATT; Nurr1 R: 3'- TAG TCA GGG TTT GCC TGG AA. The housekeeping TBP gene was used as a reference gene and its specific primer sequence is: TBP F: $5^{\prime}$ - AGG CCA GAC CCC ACA ACT C; TBP R: 3' -GGG TGG TGC CTG GCA A. The results were analyzed using the $2^{\lceil\Delta \Delta C T}$ method (Livak and Schmittgen, 2001). CT is the threshold cycle which is the number of cycles required for the fluorescent signal to cross the threshold (i.e., can be detected). CT levels are inversely proportional to the amount of target nucleic acid in the sample (i.e., the lower the CT level the greater the amount of target nucleic acid in the sample. The calibrator may be a control treatment or the sample with the highest $\Delta \mathrm{CT}$ value):

- $\quad \Delta \mathrm{CT}=\mathrm{CT}$ of the target gene "Nurr1"-CT of the reference gene "TBP"

- $\quad \Delta \Delta \mathrm{CT}=\Delta \mathrm{CT}$ of gene of interest "Nurr1"- $\Delta \mathrm{CT}$ of calibrator

\section{Results}

\section{Histoarchitecture of the Developing Mouse Forelimb}

In the present study the histological examination focused on two stages: E12.5 where the onset of cartilage formation of all limb elements appears and E14.5 where the onset of ossification takes place.

At E12.5, the proximal and distal elements of the forelimb are revealed. Sections through the forelimb show the first sign of cartilage formation as precartilage condensations of mesenchyme cells representing the presumptive upper arm (humerus), lower arm (radius and ulna) and hand plate (metacarpals) (Fig. 1A). These cells appear spherical in shape, larger in size than those of the prospective soft tissues and are randomly oriented (Fig. 1B).

At E14.5 the onset of ossification is noticed in the central part of the diaphyseal region of both upper and lower arms (Fig. 2A). Longitudinal sections through the forelimb reveal the familiar pattern of cartilage differentiation and ossification within each element (Bloom and Fawcett, 1975). In the lower arm, as well as the upper arm, the resting zone of rounded chondrocytes is observed at both ends of the radius, ulna and humerus. It is followed by larger, more flattened proliferating cells towards the central diaphysis. Then the chondrocytes become hypertrophic with calcified matrix and eventually the cells start degeneration in the region of ossification (primary ossification center) which is overlaid by a periosteal bony collar (Fig. 2B). The primary ossification center in the diaphyseal region is enclosed by a typical bilayered periosteum with an outer layer of elongated fibroblastic cells and an inner core of 2-3 cell layers thickness consisting of spherical, tightly packed and newly differentiated osteoblastic cells. These cells start secretion of the bony matrix around the peripheral chondrocytes forming a collar of osteoid matrix. In the newly formed osteoid matrix around the ossification region in the central diaphyseal area, osteoblasts become ovoid in shape and are completely surrounded by a lacunae filled with matrix forming definite osteocytes (Fig. 2C).Numerous vascular tissues are noticed in the primary ossification center. This invasion leads to cartilage resorption by osteoclasts (Fig. 2D). As the matrix is invaded by blood vessels and the hypertrophic chondrocytes are decaying, osteoblasts become closely adhere to the newly deposited osteoid matrix (Fig. 2E)

\section{Immunohistochemical Expression of Nurr1 During Forelimb Development}

To investigate the expression of Nurr1 in the developing forelimb, the same stages are chosen as in the histological study. In the limb bud at E12.5, Nurr1 is expressed only in the mesenchyme cells which will differentiate into chondrocytes. Nuclei of cells expressing Nurr1 appear brown in color (Fig. 3).

Longitudinal sections at E14.5 through the forelimb revealed that Nurr1 is highly expressed in the primary ossification centers of the elements of upper and lower arms (Fig. 4A). It is also expressed in the region of proliferating chondrocytes, hypertrophic chondrocytes and periosteum. Nuclei of cells expressing Nurr1 appear brown in color (Fig. 4B)

\section{Differential Expression Level of Nurr1 During Mouse Forelimb Development}

The expression level of Nurr1 in this study is estimated in four stages of limb development which are E10.5, E12.5, E14.5 and E18.5 using RT-PCR. Results obtained from RT-PCR analysis indicates that Nurrl is weakly expressed at stage E10.5 then the expression level increases by almost 3 folds at stage E12.5. The first significant increase was at E14.5 where it increases by almost 115 folds than that of E10.5. Nurr1 expression reaches its maximum level by stage E18.5 where it increases by almost 326 folds than that of E10.5 (Fig. 5). 

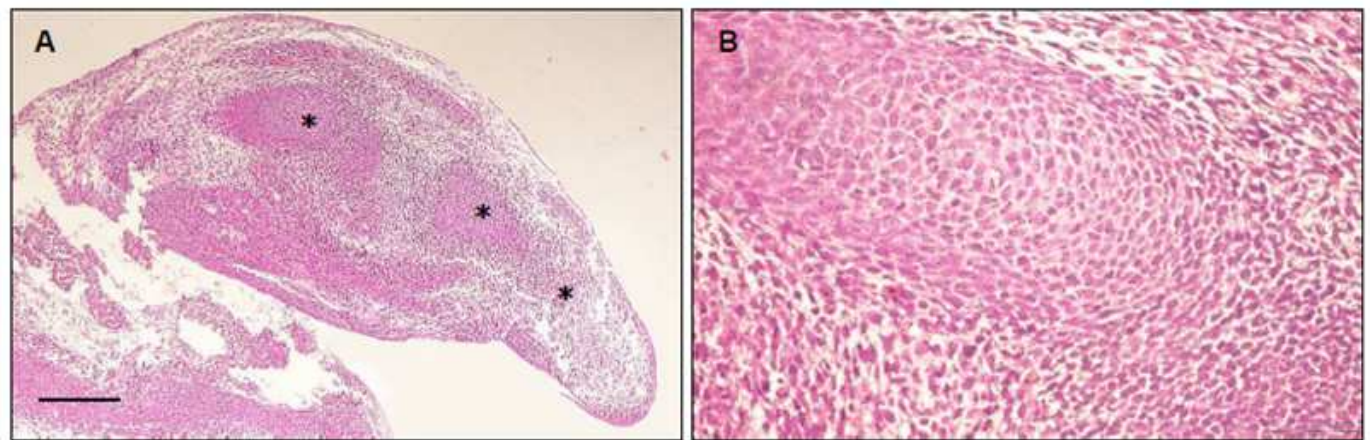

Fig. 1. Transverse section through the mouse embryo at E12.5 showing the developing forelimb bud. (A) Precartilage condensations of mesenchyme cells (asterisks) corresponding to the elements of upper and lower arms and proximal elements of the hand. (B) An enlarged part of (A) showing the precartilage condensation of mesenchyme cells in the region of the upper arm. The cells are randomly oriented and appear spherical in shape and larger in size than those of the prospective soft tissue. (A) X: 40, (B) X: 400. Scale bar represents: $2 \mathrm{~mm}$

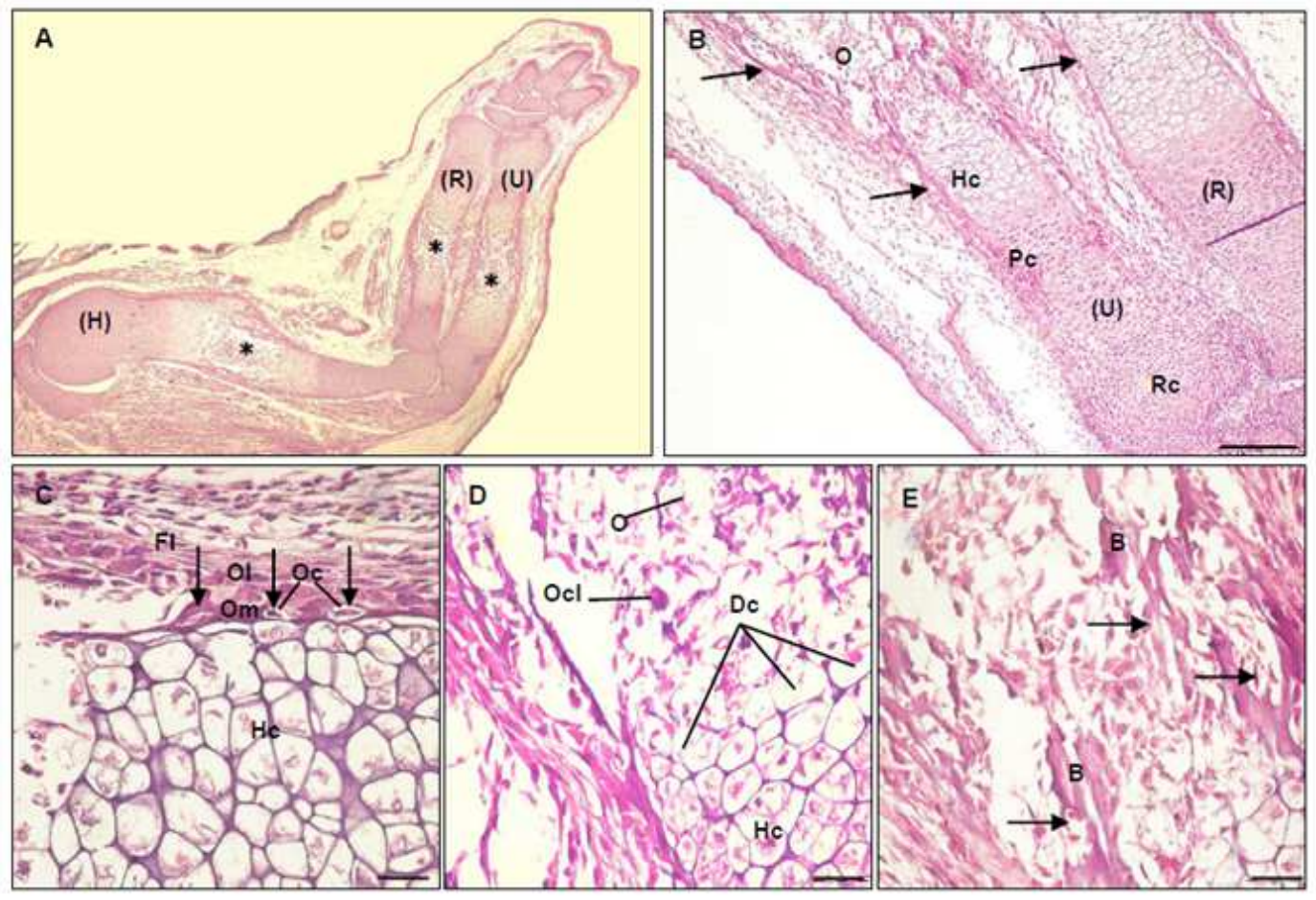

Fig. 2.(A) Longitudinal section through the mouse forelimb at E14.5. The natural curvature of the hand has resulted in the appearance of only three of five fingers. The onset of ossification (asterisks) is notable in the central part of the diaphyseal region of the elements of the upper arm and the lower arm. (B) Enlarged part of the lower arm from (A) showing the familiar pattern of endochondral ossification of radius and ulna. The rounded resting chondrocytes are followed by the more flattened proliferating chondrocytes then the hypertrophied chondrocytes and the degenerating cells in the ossification center. Bony collars (arrows) surround the area of the hypertrophied chondrocytes and the ossification center. (C) Enlarged part of (A) at the hypertrophic chondrocytes region of the radius showing the typical bilayered periosteum with an inner osteoblastic layer and an outer fibroblastic layer. The newly differentiated osteocytes are completely surrounded by lacunae (arrows) in the newly deposited osteoid matrix. (D) Enlarged part of (A) in the central diaphyseal region of the radius showing the invasion of the vascular tissue (arrow) through the primary ossification center, decaying chondrocytes and the cartilage resorption by osteoclasts. (E) Enlarged part of (A) in the central diaphyseal region of the ulna showing the newly secreted bone.The osteoblasts (arrows) which adhere closely to the osteoid matrix. H: Humerus, R: Radius, U: Ulna, Rc: Resting chondrocytes, Pc: Proliferating chondrocytes, Hc: Hypertrophied chondrocytes, Dc: Decaying chondrocytes, O: ossification center, Ol: Osteoblastic layer, Fl: Fibroblastic layer, Om: Osteoid matrix, Oc: Osteocytes, Ocl: Osteoclasts, B: Bone. (A) X: 40. (B) X: 100. (C), (D), (E) X: 400. Scale bars represent: $2 \mathrm{~mm}$ 


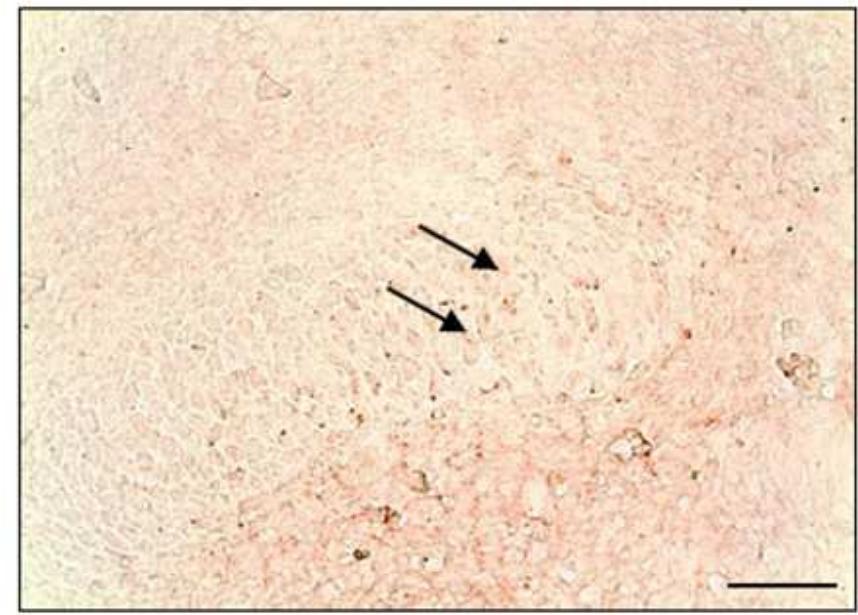

Fig. 3.Transverse section through the forelimb bud at E12.5 showing Nurr1 positive immunostaining. Nurr1 is expressed in the accumulating mesenchyme cells that will differentiate into chondrocytes (arrows). They appear brown color. X: 400 . Scale bars represent: $2 \mathrm{~mm}$
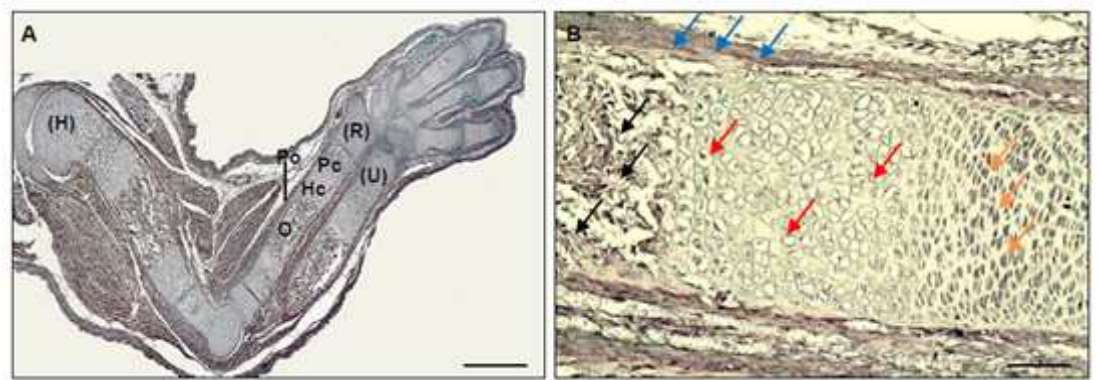

Fig. 4. Longitudinal section through the mouse forelimb at E14.5 showing Nurrl positive immunostaining. (A) Nuclei of cells expressing Nurr1 are stained in brown color, Nurrl is expressed in the primary ossification centers of humerus, radius and ulna, in proliferating chondrocytes, hypertrophic chondrocytes and periosteum. (B) Enlarged part of (A) showing Nurrl positive immunostaining in the region of the ulna. Nuclei of proliferating chondrocytes (orange arrows), hypertrophic chondrocytes (red arrows), osteoblasts in the primary ossification center (black arrows) and osteoblasts in the osteoblastic layer of periosteum (blue arrows) are stained in brown color. H: humerus, R: Radius, U: Ulna, Pc: Proliferating chondrocytes, Hc: Hypertrophic chondrocytes, O: Ossification center, Po: Periosteum. (A) X: 40, (B) X: 200. Scale bars represent: 2mm

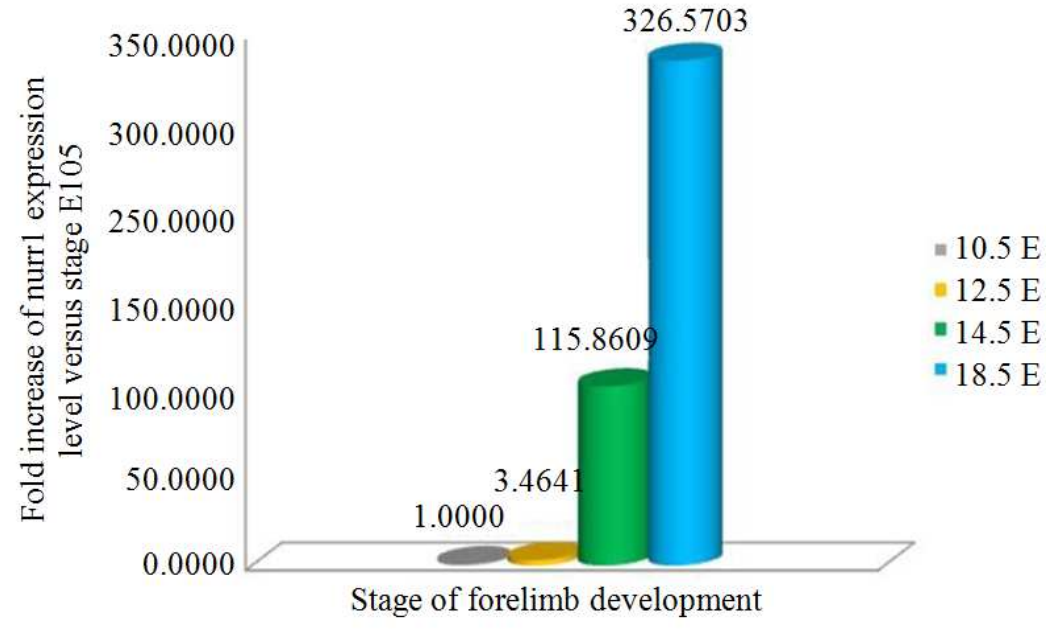

Fig. 5. A histogram showing the expression levels of Nurr1 at different stages of mouse forelimb development. The lowest expression level was at E10.5 then it increases gradually until it reaches its maximum level at E18.5 


\section{Discussion}

NR4A receptors lack identified natural ligands and as they are encoded by immediate early genes it is plausible that an important mechanism regulating their activities is to regulate their expression levels. Nurr1 has been shown to play an important role in bone homeostasis since it directly regulates Osteopontin (OPN) and OCN genes in osteoblasts (Lammi et al., 2004; Pirih et al., 2004). It has also been reported to have a protective role in cartilage homeostasis since it represses the expression of matrix metalloproteinases (which digest components of the extracellular matrix leading to the degradation of cartilage, tendon and bone) during inflammation (Mix et al., 2007). In the present study, the expression pattern of Nurr1 in the developing mouse forelimb and its relationship to limb patterning and skeletogenesis was investigated.

Nurr1 has affected both early and late osteoblast differentiation. The decreased expression of Nurr1 leads to reduced expression of osteoblast differentiation markers such as OCN and CollA1 and reduced ALP activity. The activity of these markers is also downregulated in cultured primary osteoblasts from Nurr1 null mice compared to those derived from wild type (Lee et al., 2006).

Nurr1 has also been shown to be directly induced by PTH in osteoblast in vitro (Tetradis et al., 2001a) and in calvariae and long bones in vivo (Pirih et al., 2005) suggesting that it may be a critical mediator of PTH downstream effects in osteoblasts and bone. PTH has both anabolic and catabolic effects in bone. Nurr1 is thought to be a mediator of the anabolic effects of short term PTH treatment (Lammi, 2008) since the short, intermittent PTH treatment activate bone formation but doesn't have time to activate bone resorption.

Although Nurr1 has been reported in the developing mouse hindlimbs at E11.5 (Zetterström et al., 1996b), its role in bone development has remained elusive since The skeletal phenotype of Nurr1 knockout mice has not been previously examined due to the death of Nurr1-deficient mice within $24 \mathrm{~h}$ after birth (Lammi et al., 2004). Our study is the first to investigate the expression pattern of Nurr1 in the developing mouse forelimb and its relationship to skeletogenesis and osteogenesis.

We reported that Nurr1 is expressed in the developing limb at E12.5 in the mesenchyme cells that will form the skeletal elements of the forelimb implying that Nurr1 plays a role at the onset of skeletogenesis. It is also expressed in the proliferating chondrocytes, the hypertrophic chondrocytes and the osteoblasts of the primary ossification centers of the forelimb skeletal elements at E14.5 suggesting that it has an important role in bone development and bone remodeling. This assumption is consistent with our results from RT-PCR analysis of Nurr1 expression levels in the developing mouse forelimb. These data revealed that Nurr1 expression level increases progressively during limb development and that it is highly increased by E14.5 at the onset of ossification and reached its peak of expression at E18.5 where the continuation of ossification and bone elongation occur. These results were demonstrated by RT-PCR and confirmed by immunohistochemical assay in our study.

Crosstalk between Nurr1 and some signaling pathways in osteoblasts were reported. Nurr1 were found to repress both Wnt signaling pathway and $\beta$-catenin signaling pathway in osteoblasts (Rajalin and Aarnisalo, 2011). Nurr1 was also found to be stimulated by FGF-8 in osteoblasts (Lammi and Aarnisalo, 2008). Whether these crosstalks are also present in the developing limb remain elusive and need further investigation.

In summary, our results thus add to the previous data on the role of Nurr1 in the development and function of bone tissue. However, further studies to elucidate the potential contribution of Nurr1 in the bone physiopathological conditions remain to be addressed.

\section{Acknowledgment}

We are grateful to Frederic Brau (microscope imaging) and Nathalie Leroudier (RT-qPCR) at the Molecular and Cellular Pharmacological Institute in France for their technical assistance.

\section{Author's Contributions}

Heba A. Ahmed: Collected the forelimbs from the animals, performed most of the histological experiments, carried out the acquisition and/or analyses of most of the data and wrote the manuscript.

Lidia L. Ibrahim: Supervised the project, carried out the analyses of data and revised the manuscript.

Desouki A. El Mekkawy: Supervised the project, carried out the analyses of data and revised the manuscript.

Abeer EI Wakil: Performed the immunohistochemical and molecular experiments and analyses, designed the study, wrote and revised the manuscript.

\section{Ethics}

All experimental protocols were approved by the local council for animal care and were conducted according to Egyptian laws for animal experimentation.

\section{Conflict of Interest Statement}

The authors have no conflicts of interest to disclose.

\section{References}

Bloom, W. and D.W. Fawcett, 1975. A Textbook of Histology. 10th Eds., Saunders, Philadelphia, pp: 616. 
Castillo, S.O., J.S. Baffi, M. Palkovits, D.S. Goldstein and I.J. Kopin et al., 1998. Dopamine biosynthesis is selectively abolished in substantia nigra/ventral tegmental area but not in hypothalamic neurons in mice with targeted disruption of the Nurr1 gene. Molecular Cellular Neurosciences, 11: 36-46. DOI: 10.1006/mcne.1998.0673

Chomczynski, P. and N. Sacchi, 2006. The single-step method of RNA isolation by acid guanidinium thiocyanate-phenol-chloroform extraction: Twentysomething years on. Nature Protocols, 1: 581-585. DOI: 10.1038 /nprot.2006.83

Flaig, R., H. Greschik, C. Peluso-Iltis and D. Moras, 2005. Structural basis for the cell-specific activities of the NGFI-B and the Nurr1 ligand-binding domain. J. Biol. Chemistry, 280: 19250-8. DOI: $10.1074 /$ jbc.M413175200

Giguére, V., 1999. Orphan nuclear receptors: From gene to function. Endocrine Rev., 20: 689-725. DOI: $10.1210 /$ edrv.20.5.0378

Honkaniemi, J., J.S. Zhang, F.M. Longo and F.R. Sharp, 2000. Stress induces zinc finger immediate early genes in the rat adrenal gland. Brain Res., 877: 203208. DOI: $10.1016 / \mathrm{S} 0006-8993(00) 02673-1$

Lammi, J., J. Huppunen and P. Aarnisalo, 2004. Regulation of the osteopontin gene by the orphan nuclear receptor NURR1 in osteoblasts. Molecular Endocrinol. (Baltimore, Md.), 18: 1546-57. DOI: 10.1210/me.2003-0247

Lammi, J., 2008. Orphan Nuclear Receptor Subfamily NR4A: Their Interplay with Other Nuclear Receptors and Functions in Osteoblasts. 1st Edn., Institute of Biomedicine/Physiology, Helsinki, Finland.

Lammi, J. and P. Aarnisalo, 2008. FGF-8 stimulates the expression of NR4A orphan nuclear receptors in osteoblasts. Molecular Cellular Endocrinol., 295: 87-93. DOI: 10.1016/j.mce.2008.08.023

Laudet, V. and H. Gronemeyer, 2002. The nuclear receptor factsbook, Issue 964 . Academic press.

Law, S.W., O.M. Conneely, F.J. DeMayo and B.W. O'Malley, 1992. Identification of a new brainspecific transcription factor, NURR1. Molecular Endocrinology (Baltimore, Md.), 6: 2129-35. DOI: 10.1210/mend.6.12.1491694

Lee, M.K., H. Choi, M. Gil and V.M. Nikodem, 2006. Regulation of osteoblast differentiation by Nurr1 in MC3T3-E1 cell line and mouse calvarial osteoblasts. J. Cellular Biochemistry, 99: 986-94. DOI: $10.1002 /$ jcb. 20990

Liu, D., H. Jia, D.I.R. Holmes, A. Stannard and I. Zachary, 2003. Vascular endothelial growth factorregulated gene expression in endothelial cells: KDRmediated induction of Egr3 and the related nuclear receptors Nur77, Nurr1 and Nor1. Arteriosclerosis, Thrombosis Vascular Biol., 23: 2002-7. DOI: 10.1161/01.ATV.0000098644.03153.6F
Livak, K.J. and T.D. Schmittgen, 2001. Analysis of relative gene expression data using real-time quantitative PCR and the $2^{-\Delta \Delta C T}$ method. Methods 25: 402-8. DOI: $10.1006 /$ meth.2001.1262

Milbrandt, J., 1988. Nerve growth factor induces a gene homologous to the glucocorticoid receptor gene. Neuron, 1: 183-8.

DOI: $10.1016 / 0896-6273(88) 90138-9$

Mix, K.S., M.G. Attur, H. Al-Mussawir, S.B. Abramson and C.E. Brinckerhoff et al., 2007. Transcriptional repression of matrix metalloproteinase gene expression by the orphan nuclear receptor NURR1 in cartilage. J. Biol. Chemistry, 282: 9492-504. DOI: $10.1074 / j b c . M 608327200$

Murphy, E.P., A. McEvoy, O.M. Conneely, B. Bresnihan and O. FitzGerald, 2001. Involvement of the nuclear orphan receptor NURR1 in the regulation of corticotropin-releasing hormone expression and actions in human inflammatory arthritis. Arthritis Rheumatism, 44: 782-93.

Ohkura, N., M. Ito, T. Tsukada, K. Sasaki and K. Yamaguchi et al., 1996. Structure, mapping and expression of a human NOR-1 gene, the third member of the Nur77/NGFI-B family. Biochimica et Biophysica Acta (BBA) - Gene Structure Expression, 1308: 205-214. DOI: $10.1016 / 0167-4781(96) 00101-7$

Ohkubo, T., Y. Sugawara, K. Sasaki, K. Maruyama and N. Ohkura et al., 2002. Early induction of nerve growth factor-induced genes after liver resectionreperfusion injury. J. Hepatol., 36: 210-217. DOI: $10.1016 / \mathrm{S} 0168-8278(01) 00258-6$

Pirih, F.Q., J.M. Nervina, L. Pham, T. Aghaloo and S. Tetradis, 2003. Parathyroid hormone induces the nuclear orphan receptor NOR-1 in osteoblasts. Biochemical Biophysical Res. Commun., 306: 14450. DOI: 10.1016/S0006-291X(03)00931-8

Pirih, F., A. Tang and I. Ozkurt, 2004. Nuclear orphan receptor Nurr1 directly transactivates the osteocalcin gene in osteoblasts. J. Biological Chemistry, 279: 53167-53174. DOI: 10.1074/jbc.M405677200

Pirih, F.Q., T.L. Aghaloo, O. Bezouglaia, J.M. Nervina and S. Tetradis, 2005. Parathyroid hormone induces the NR4A family of nuclear orphan receptors in vivo. Biochemical Biophysical Res. Commun., 332: 494-503. DOI: 10.1016/j.bbrc.2005.04.132

Rajalin, A.M. and P. Aarnisalo, 2011. Cross-talk between NR4A orphan nuclear receptors and $\beta$ catenin signaling pathway in osteoblasts. Archives Biochemistry Biophysics, 509: 44-51.

DOI: 10.1016/j.abb.2011.02.018

Saucedo-Cardenas, O. and O.M. Conneely, 1996. Comparative distribution of $\mathrm{NURR}_{1}$ and NUR77 nuclear receptors in the mouse central nervous system. J. Molecular Neuroscience MN, 7: 51-63. DOI: $10.1007 / \mathrm{BF} 02736848$ 
Smirnov, A.N., 2002. Nuclear receptors: nomenclature, ligands, mechanisms of their effects on gene expression. Biochemistry Biokhimiia, 67: 957-977. DOI: 10.1023/A:1020545200302

Tetradis, S., O. Bezouglaia and A. Tsingotjidou, 2001a. Parathyroid hormone induces expression of the nuclear orphan receptor Nurrl in bone cells. Endocrinol., 142: 663-70. PMID: 11159837

Tetradis, S., O. Bezouglaia, A. Tsingotjidou and A. Vila, 2001b. Regulation of the nuclear orphan receptor Nur77 in bone by parathyroid hormone. Biochemical Biophysical Res. Commun., 281: 913-6. DOI: $10.1006 /$ bbrc.2001.4459

Wang, Z., G. Benoit, J. Liu, S. Prasad and P. Aarnisalo et al., 2003. Structure and function of Nurr1 identifies a class of ligand-independent nuclear receptors. Nature, 423: 555-60.
Zetterström, R.H., L. Solomin, T. Mitsiadis, L. Olson and T. Perlmann, 1996a. Retinoid X receptor heterodimerization and developmental expression distinguish the orphan nuclear receptors NGFI-B, Nurr1 and Nor1. Molecular Endocrinol., 10: 165666. DOI: $10.1210 /$ mend.10.12.8961274

Zetterström, R.H., R. Williams, T. Perlmann and L. Olson, 1996b. Cellular expression of the immediate early transcription factors Nurr1 and NGFI-B suggests a gene regulatory role in several brain regions including the nigrostriatal dopamine system. Brain Res. Molecular Brain Res., 41: 111-20.

DOI: 10.1016/0169-328X(96)00074-5

Zetterström, R.H., L. Solomin, L. Jansson, B.J. Hoffer and L. Olson et al., 1997. Dopamine neuron agenesis in Nurr1-deficient mice. Sci. 276: 248-50. DOI: $10.1126 /$ science 276.5310 .248 